\title{
Constraints on Heavy Neutrino and SUSY Parameters Derived from the Study of Neutrinoless Double Beta Decay
}

\author{
Andrei Neacsu ${ }^{1,2}$ and Sabin Stoica ${ }^{1,2}$ \\ ${ }^{1}$ Horia Hulubei Foundation, P.O. Box MG-12, Magurele, Bucharest 077125, Romania \\ ${ }^{2}$ Horia Hulubei National Institute for Physics and Nuclear Engineering, P.O. Box MG-6, Magurele, \\ Bucharest 077125, Romania \\ Correspondence should be addressed to Andrei Neacsu; nandrei@theory.nipne.ro
}

Received 4 April 2014; Accepted 26 May 2014; Published 16 June 2014

Academic Editor: Theocharis Kosmas

Copyright ( 2014 A. Neacsu and S. Stoica. This is an open access article distributed under the Creative Commons Attribution License, which permits unrestricted use, distribution, and reproduction in any medium, provided the original work is properly cited. The publication of this article was funded by SCOAP ${ }^{3}$.

\begin{abstract}
New constraints on the lepton number violating (LNV) parameters are derived from the analysis of the neutrinoless double beta $(0 \nu \beta \beta)$ decay in the hypothesis that this process would occur through the exchange of heavy neutrinos and/or SUSY particles. For derivation, we use new values of both phase space factors (PSFs) and nuclear matrix elements (NMEs) calculated with numerical codes developed recently, as well as the most recent experimental lifetimes. The NMEs are computed with a shell model (ShM) code for ${ }^{48} \mathrm{Ca},{ }^{76} \mathrm{Ge}$, and ${ }^{82} \mathrm{Se}$ nuclei, while at present similar ShM results are available only for the first nucleus. We compare our results with similar ones from literature, obtained with ShM, QRPA, and IBM-2 methods, and conclude that more results are needed for a relevant analysis on the validity of NMEs associated with these decay mechanisms.
\end{abstract}

\section{Introduction}

Neutrinoless double beta $(0 \nu \beta \beta)$ decay is a beyond standard model (BSM) process by which an even-even nucleus transforms into another even-even nucleus with the emission of two electrons/positrons but no antineutrinos/neutrinos in the final states. Its study is important since it would clarify the question about the lepton number violation, decide on the neutrinos character (are they distinguished or not from their antiparticles?), and give a hint on the scale of their absolute masses. Moreover, the study of $0 \nu \beta \beta$ decay has a broader potential to search for other BSM phenomena, such as new neutrino flavors and exotic particles. The great interest in understanding these fundamental issues has led to many theoretical and experimental investigations of this process. The reader can find up-to-date information on these studies in several recent reviews [1-6], which also contain therein a comprehensive list of references in the domain.

One of the open issues concerning the $0 \nu \beta \beta$ decay is related to the possible mechanisms responsible for its occurrence. The exchange of a light Majorana neutrino in the presence of left handed (LH) weak interaction is still the most popular and studied mechanism. However, other mechanisms have also been considered, for example, the exchange of heavy neutrinos $[7,8]$ and SUSY particles $[3,9]$, which could also contribute to the total $0 \nu \beta \beta$ decay rate. On the other hand, constraints on heavy neutrino and SUSY parameters can be now derived at hadron colliders, as well, by analyzing same sign dilepton decay channels, which are triggered by processes that violate the lepton number conservation by two units (like $0 \nu \beta \beta$ decay). Indeed, the CMS, ATLAS, and LHCb experiments at LHC include now in their data analysis the search of such channels, and the first results were already reported [10-13]. Thus, complementary information that allows the constraint of various LNV parameters can also be obtained now from high-energy experiments.

For all mechanisms, the $0 \nu \beta \beta$ lifetimes can be expressed in a good approximation as a product of a phase space factor, a nuclear matrix element related to the nuclear structure of the parent and daughter nuclei, and a LNV parameter related to the BSM mechanism considered. These three factors can be identified in (1) and (2) from the next section. Hence, to 
extract reliable upper limits for the LNV parameters, we need accurate calculation of both PSFs and NMEs factors, as well as reliable measurements of the lifetimes.

The largest uncertainties in the theoretical calculations for double beta decay (DBD) arise from the calculated values of the NMEs. For the LH light neutrino exchange mechanism, the NMEs are currently computed by several methods, the present most employed ones being the proton-neutron quasirandom phase approximation (pnQRPA) [14-20], interacting shell model (ISM) [21-25], interacting boson model (IBM) [26-28], projected Hartree Fock Bogoliubov (PHFB) [29], and energy density functional (EDS) [30] methods. At present, there are still large discrepancies between the NMEs values computed with different methods and by different groups, which have been widely discussed in literature (see, e.g., $[5,6])$ for the light neutrino exchange mechanism. At present, for heavy neutrino and SUSY exchange mechanisms, there are fewer NME calculations, performed with QRPA $[31,32]$ and IBA-2 [28] and ShM methods [33, 34]. The discrepancies between the existing NME values associated with heavy neutrino or SUSY mechanisms are even larger than those in the case of LH light neutrino exchange, so there is a need for new calculations.

In this paper, we report new constraints on the heavy neutrino and SUSY parameters derived from the analysis of the neutrinoless double beta decay of three experimentally interested nuclei, ${ }^{48} \mathrm{Ca},{ }^{76} \mathrm{Ge}$, and ${ }^{82} \mathrm{Se}$, in the hypothesis that this process would occur through the exchange of such particle. The computation of the NMEs is performed with a ShM code developed recently and presented in detail in $[35,36]$. Also, we estimate the uncertainties introduced in calculations by the use of different $\mathrm{NN}$ interactions and SRC parameterizations and found that the calculated NME values depend significantly on these nuclear ingredients. We compare our results with other previous ones from literature performed with ShM, QRPA, and IBM-2 approaches and discuss the discrepancies. We remark that, until now, large scale ShM calculations of NMEs corresponding to these mechanisms are available only for ${ }^{48} \mathrm{Ca}$, and they differ significantly from the IBM-2 calculations. In order to understand these discrepancies, more ShM calculations of the NMEs for these (less discussed) $0 \nu \beta \beta$ decay mechanisms, including other nuclei, are needed.

The values of the PSFs are taken from our recent work [37], which are very close to those reported in [38, 39]. We mention that PSFs for DBD have been calculated since long time [40, 41], but they were less discussed, being considered to be computed with enough precision. Recently, they were recalculated with improved approaches $[38,39,42]$ and several discrepancies were revealed as compared to the old calculations, which should be taken into account for accurate predictions of $0 \nu \beta \beta$ lifetimes and derivation of LNV parameters.

The paper is organized as follows. In the next section, we present the formulae for the $0 \nu \beta \beta$ decay lifetimes together with the relevant expressions for the PSFs and NMEs which have to be computed for deriving the LNV parameters, for heavy neutrino and SUSY mechanisms. In Section 3, we present and discuss our results, while in the last section we formulate the conclusions of our work.

\section{Formalism}

In this section, we give a short description of the heavy neutrino and SUSY formalisms, displaying the PSF and NME formulas that have to be computed. As we already mentioned, the expressions of the $0 \nu \beta \beta$ lifetimes can be written as a product of three terms: a phase space, a nuclear matrix elements, and a term related to the corresponding LNV mechanism:

$$
\left(T_{1 / 2}^{0 \nu}\right)^{-1}=G^{0 v}\left(Q_{\beta \beta}, Z\right) \sum_{k}\left(\left|M_{k}^{0 v}\right|^{2}\right)\left(\eta_{k}\right)^{2},
$$

where $k$ is an index which denotes the mechanisms that can contribute to the $0 \nu \beta \beta$ decay. In this work, we refer to the heavy neutrino and SUSY exchange mechanisms, so the lifetime reads

$$
\begin{aligned}
\left(T_{1 / 2}^{0 \nu}\right)^{-1}=G^{0 \nu}\left(Q_{\beta \beta}, Z\right) & \left(\left|M_{N}^{0 v}\right|\left\langle\eta_{N}\right\rangle+\left|M_{\lambda^{\prime}}^{0 \nu}\right|\left\langle\eta_{\lambda^{\prime}}\right\rangle\right. \\
& \left.+\left|M_{q}^{0 v}\right|\left\langle\eta_{q}\right\rangle\right)^{2}
\end{aligned}
$$

where $G^{0 \nu}$ is the phase space factor for this decay mode, depending on the energy decay $Q_{\beta \beta}$ and nuclear charge $Z$. $M_{N, \lambda^{\prime}, q}^{0 v}$ are the NMEs associated with the heavy neutrino $(N)$ exchange mechanism and with the gluino $\left(\lambda^{\prime}\right)$ and squarkneutrino $(q)$ exchange mechanisms. The last mechanisms may appear in SUSY theories with R-parity violation [3]. $\eta_{N, \lambda^{\prime}, q}$ are the corresponding coupling parameters associated with these mechanisms.

For the $0 \nu \beta \beta$ decay, the expression of the PSFs reads

$$
G_{0 \nu}=\frac{2}{4 g_{A}^{4} R^{2} \ln 2} \int_{m_{e} c^{2}}^{T_{0}-m_{e} c^{2}} f_{11}^{(0)} w_{0 \nu} d \epsilon_{1}
$$

where $f_{11}^{(0)}$ are the solutions of the Dirac equation and

$$
w_{0 \nu}=\frac{g_{A}^{4}\left(G \cos \theta_{C}\right)^{4}}{16 \pi^{5}}\left(m_{e} c^{2}\right)^{2}\left(\hbar c^{2}\right)\left(p_{1} c\right)\left(p_{2} c\right) \epsilon_{1} \epsilon_{2}
$$

where $g_{A}\left(g_{A}=1.25\right)$ is the axial-vector coupling constant, $G=1.16637 \times 10^{-5} \mathrm{GeV}^{-2}$ is the Fermi constant, $\theta_{C}$ is the Cabbibo angle, $m_{e}$ is the electron rest mass, while $p_{1}, p_{2}$ and $\epsilon_{1}, \epsilon_{2}$ are the electron momenta and energies, respectively. In (3), it is convenient to redefine the PSFs by a renormalization that eliminates the constant $g_{A}$ and correlates (by dividing by $4 R^{2}, R$ the nuclear radius) the dimension of $G_{0 v}$ with the NMEs which are dimensionless, such that the PSFs are expressed in $\left[\mathrm{yr}^{-1}\right]$.

Concerning the NMEs, their expressions can be written, in general, as a sum of three components:

$$
M^{0 v}=M_{\mathrm{GT}}^{0 v}-\left(\frac{g_{V}}{g_{A}}\right)^{2} \cdot M_{F}^{0 v}-M_{T}^{0 v},
$$

where $M_{\mathrm{GT}}^{0 \nu}, M_{F}^{0 \nu}$, and $M_{T}^{0 \nu}$ are the Gamow-Teller (GT), Fermi $(F)$, and Tensor $(T)$ components, respectively. 
Due to the two-body nature of the transition operator, the NMEs can be expressed as a sum of products of two-body transition densities (TBTDs) and matrix elements of the twobody transition operators for two-particle states, shortly, twobody matrix elements (TBMEs) [35, 36]. Consider

$$
\begin{aligned}
M_{\alpha}^{0 v}=\sum_{j_{p} j_{p^{\prime}} j_{n} j_{n^{\prime}} J_{\pi}} \operatorname{TBTD}\left(j_{p} j_{p^{\prime}}, j_{n} j_{n^{\prime}} ; J_{\pi}\right) \\
\times\left\langle j_{p} j_{p^{\prime}} ; J_{\pi}\left\|\tau_{-1} \tau_{-2} O_{12}^{\alpha}\right\| j_{n} j_{n^{\prime}} ; J_{\pi}\right\rangle,
\end{aligned}
$$

where $\left|j j^{\prime} ; J^{\pi}\right\rangle$ represent the antisymmetrized two-particle states and $O_{m n}^{\alpha}$ are DBD transition operators $(\alpha=\mathrm{GT}, F, T)$. Since the NN effective interaction can be treated by means of a central (single-particle) potential, the NMEs can be conveniently calculated using Moshinsky's transformations between the relative and center of mass (CM) coordinates and the proper use of nuclear states in different coupling notations. The calculation of the matrix elements of these operators can be decomposed into products of reduced matrix elements within the spin and relative coordinates subspaces [21,35]. The expressions of the two-body transition operators are

$$
\begin{aligned}
& O_{12}^{\mathrm{GT}}=\sigma_{1} \cdot \sigma_{2} H(r), \quad O_{12}^{F}=H(r), \\
& O_{12}^{T}=\sqrt{\frac{2}{3}}\left[\sigma_{1} \times \sigma_{2}\right]^{2} \cdot \frac{r}{R} H(r) C^{(2)}(\widehat{r}),
\end{aligned}
$$

where $\sigma$ are the Pauli spin operators and $C^{(2)}(\widehat{r})$ is the center of mass operator. The expressions for the neutrino potentials $H_{\alpha}$ in the case of heavy neutrino mechanisms are

$$
H_{\alpha}(r)=\frac{2 R}{\pi m_{e} m_{p}} \int_{0}^{\infty} j_{k}(q r) h_{\alpha}\left(q^{2}\right) q^{2} d q,
$$

where $m_{p}$ is the proton mass and $j_{k}(k=0$ for $\alpha=\mathrm{GT}, F$, and $k=2$ for $\alpha=T$ ) are the Bessel spherical functions. The expressions of $h_{\alpha}(\alpha=\mathrm{GT}, F, T)$ are

$$
\begin{aligned}
& h_{F}\left(q^{2}\right)=G_{V}^{2}\left(q^{2}\right), \\
& h_{\mathrm{GT}}\left(q^{2}\right)= \frac{G_{A}^{2}\left(q^{2}\right)}{g_{A}^{2}}\left[1-\frac{2}{3} \frac{q^{2}}{q^{2}+m_{\pi}^{2}}+\frac{1}{3}\left(\frac{q^{2}}{q^{2}+m_{\pi}^{2}}\right)^{2}\right] \\
&+\frac{2}{3} \frac{G_{M}^{2}\left(q^{2}\right)}{g_{A}^{2}} \frac{q^{2}}{4 m_{p}^{2}}, \\
& h_{T}\left(q^{2}\right)= \frac{G_{A}^{2}\left(q^{2}\right)}{g_{A}^{2}}\left[\frac{2}{3} \frac{q^{2}}{q^{2}+m_{\pi}^{2}}-\frac{1}{3}\left(\frac{q^{2}}{q^{2}+m_{\pi}^{2}}\right)^{2}\right] \\
&+\frac{1}{3} \frac{G_{M}^{2}\left(q^{2}\right)}{g_{A}^{2}} \frac{q^{2}}{4 m_{p}^{2}},
\end{aligned}
$$

where $m_{\pi}$ is the pion mass and

$$
G_{M}\left(q^{2}\right)=\left(\mu_{p}-\mu_{n}\right) G_{V}\left(q^{2}\right)
$$

with $\left(\mu_{p}-\mu_{n}\right)=4.71$. The expressions (9)-(11) include the following nuclear effects: (i) finite nucleon size (FNS) effects through the nucleon form factors $G_{A}, G_{V}$ and (ii) higher order current (HOC) effects by the second and third terms in the $h_{\mathrm{GT}}$ expression and by the appearance of the $h_{T}$ contribution.

The $G_{V}$ and $G_{A}$ form factors which take into account the finite size of the nucleons effect are

$$
\begin{aligned}
& G_{A}\left(q^{2}\right)=g_{A}\left(\frac{\Lambda_{A}^{2}}{\Lambda_{A}^{2}+q^{2}}\right)^{2}, \\
& G_{V}\left(q^{2}\right)=g_{V}\left(\frac{\Lambda_{V}^{2}}{\Lambda_{V}^{2}+q^{2}}\right)^{2} .
\end{aligned}
$$

For the vector and axial coupling constants, the majority of calculations take $g_{V}=1$ and the unquenched value $g_{A}=$ 1.25 , while the values of the vector and axial vector form factors are $\Lambda_{V}=850 \mathrm{MeV}$ and $\Lambda_{A}=1086 \mathrm{MeV}$ [1], respectively.

For the SUSY mechanisms, only the GT and $T$ components of the $M^{0 v}$ are contributing. For the gluino exchange mechanism (SUSY1), the radial neutrino potentials $H_{\alpha}$ have a similar form as that for the heavy neutrino mechanism (9), but with different $h_{\alpha}$ factors $[3,14]$ :

$$
\mathscr{M}_{\lambda^{\prime}}^{0 \gamma}=c^{1 \pi}\left(M_{\mathrm{GT}}^{1 \pi}-M_{T}^{1 \pi}\right)+c^{2 \pi}\left(M_{\mathrm{GT}}^{2 \pi}-M_{T}^{2 \pi}\right),
$$

with

$$
\begin{aligned}
c^{1 \pi} & =-\frac{2}{9} \frac{\sqrt{2} f_{\pi} m_{\pi}^{4}}{m_{p}^{3} m_{e}\left(m_{u}+m_{d}\right)} \frac{g_{s} F_{P}}{g_{A}^{2}}, \\
c^{2 \pi} & =\frac{1}{18} \frac{f_{\pi}^{2} m_{\pi}^{4}}{m_{p}^{3} m_{e}\left(m_{u}+m_{d}\right)^{2}} \frac{g_{s}^{2}}{g_{A}^{2}},
\end{aligned}
$$

where $m_{\pi}$ is the charged-pion mass, $139.6 \mathrm{MeV}, f_{\pi}=$ $0.668 m_{\pi} \mathrm{MeV}, m_{u}$ and $m_{d}$ are the up and down quark masses $\left(m_{u}+m_{d}\right)=11.6 \mathrm{MeV}, m_{p}=938.27 \mathrm{MeV}, m_{e}=0.511 \mathrm{MeV}$, $g_{s}=13.4$, and $F_{P} \approx 4.41$ [3]. Computing the expressions from (15), we obtain $c^{1 \pi}=-85.33$ and $c^{2 \pi}=365.72$. Consider

$$
\begin{aligned}
& H_{\alpha}^{1 \pi}(r)=-\frac{2 R}{\pi} \int_{0}^{\infty} j_{k}(q r) h_{\alpha}^{1 \pi}\left(q^{2}\right) d q, \\
& H_{\alpha}^{2 \pi}(r)=-\frac{4 R}{\pi} \int_{0}^{\infty} j_{k}(q r) h_{\alpha}^{2 \pi}\left(q^{2}\right) d q,
\end{aligned}
$$

with

$$
\begin{gathered}
h_{\alpha}^{1 \pi}\left(q^{2}\right)=\frac{G_{A}^{2}\left(q^{2}\right)}{g_{A}^{2}} \frac{q^{4} / m_{\pi}^{4}}{1+q^{2} / m_{\pi}^{2}}, \\
h_{\alpha}^{2 \pi}\left(q^{2}\right)=\frac{G_{A}^{2}\left(q^{2}\right)}{g_{A}^{2}} \frac{q^{4} / m_{\pi}^{4}}{\left(1+q^{2} / m_{\pi}^{2}\right)^{2}} .
\end{gathered}
$$

For the squark-neutrino mechanism (SUSY2), $H_{\alpha}$ has an expression similar to the light neutrino mechanism:

$$
H_{\alpha}(r)=\frac{2 R}{\pi} \int_{0}^{\infty} j_{k}(q r) \frac{h_{\alpha}\left(q^{2}\right)}{q+\langle E\rangle} G_{\alpha}\left(q^{2}\right) q d q,
$$


TABLE 1: $0 \nu \beta \beta$ NMEs values for heavy neutrino mechanism and comparison to other results in the literature.

\begin{tabular}{lcccc}
\hline & No SRC & Jastrow & AV-18 & CD-BONN \\
\hline${ }^{48} \mathrm{Ca}(\mathrm{GXPF1A})$ & 91.5 & 23.7 & 47.5 & 70.3 \\
${ }^{48} \mathrm{Ca}(\mathrm{KB} 3 \mathrm{G})$ & 106.8 & 29.7 & 56.8 & 82.8 \\
${ }^{48} \mathrm{Ca}[34]$ & & & 52.9 & 75.5 \\
${ }^{48} \mathrm{Ca}[28]$ & & 16.3 & 46.3 & 76.0 \\
${ }^{76} \mathrm{Ge}(\mathrm{JUN} 45)$ & 255.7 & 73.1 & 137.8 & 199.2 \\
${ }^{76} \mathrm{Ge}[31]^{\mathrm{a}},[32]^{\mathrm{b}}$ & & $32.6^{\mathrm{a}}$ & $233^{\mathrm{b}}$ & $351^{\mathrm{b}}$ \\
${ }^{76} \mathrm{Ge}[28]$ & & 48.1 & 107 & 163 \\
${ }^{82} \mathrm{Se}(\mathrm{JUN} 45)$ & 237.3 & 66.1 & 126.9 & 184.5 \\
${ }^{82} \mathrm{Se}[31]^{\mathrm{a}},[32]^{\mathrm{b}}$ & & $30.0^{\mathrm{a}}$ & $226^{\mathrm{b}}$ & $340^{\mathrm{b}}$ \\
${ }^{82} \mathrm{Se}[28]$ & & 35.6 & 84.4 & 132 \\
\hline
\end{tabular}

The indices $\mathrm{a}$ and $\mathrm{b}$ are used to indicate different calculations performed by the Tubingen group.

TABLE 2: $0 \nu \beta \beta$ NMEs values for SUSY1 mechanism and comparison to other results in the literature.

\begin{tabular}{lcccc}
\hline & No SRC & Jastrow & AV-18 & CD-BONN \\
\hline${ }^{48} \mathrm{Ca}(\mathrm{GXPF1A})$ & 643.7 .2 & 242.4 & 417.2 & 548.2 \\
${ }^{48} \mathrm{Ca}(\mathrm{KB} 3 \mathrm{G})$ & 750.1 & 293.3 & 492.47 & 641.7 \\
${ }^{48} \mathrm{Ca}[34]$ & & & 453 & 618 \\
${ }^{48} \mathrm{Ca}[43]$ & 392 & 147 & & \\
\hline${ }^{76} \mathrm{Ge}(\mathrm{JUN} 45)$ & 1762.1 & 678.3 & 1156.2 & 1509.4 \\
${ }^{76} \mathrm{Ge}[43]^{\mathrm{a}},[32]^{\mathrm{b}}$ & $1831^{\mathrm{a}}$ & $625^{\mathrm{a}}$ & $587^{\mathrm{b}}$ & $515^{\mathrm{b}}$ \\
${ }^{82} \mathrm{Se}(\mathrm{JUN} 45)$ & 1628.0 & 612.8 & 1062.5 & 1393.5 \\
${ }^{82} \mathrm{Se}[43]^{\mathrm{a}},[32]^{\mathrm{b}}$ & $1667^{\mathrm{a}}$ & $583^{\mathrm{a}}$ & $574^{\mathrm{b}}$ & $504^{\mathrm{b}}$ \\
\hline
\end{tabular}

The indices $\mathrm{a}$ and $\mathrm{b}$ are used to indicate different calculations performed by the Tubingen group.

where $\langle E\rangle$ is the energy used in the closure approximation and represents the average excitation energy of the states in the intermediate odd-odd nucleus that contribute to the decay. The $h_{\alpha}$ factors have in this case the following expressions $[3,14]$ :

$$
h_{\mathrm{GT}, T}=-\frac{1}{6} \frac{m_{\pi}^{2}}{m_{e}\left(m_{u}+m_{d}\right)} \frac{q^{2} / m_{\pi}^{2}}{\left(1+q^{2} / m_{\pi}^{2}\right)^{2}} .
$$

For computing the radial matrix elements $\left\langle n l\left|H_{\alpha}\right| n^{\prime} l^{\prime}\right\rangle$, we use the harmonic oscillator wave functions $\psi_{n l}(l r)$ and $\psi_{n^{\prime} l^{\prime}}(r)$ corrected by a factor $[1+f(r)]$, which takes into account the SRCs induced by the nuclear interaction:

$$
\psi_{n l}(r) \longrightarrow[1+f(r)] \psi_{n l}(r) .
$$

For the correlation function, we take the functional form

$$
f(r)=-c \cdot e^{-a r^{2}}\left(1-b r^{2}\right),
$$

where $a, b$, and $c$ are constants which have particular values in different parameterizations. In this work, we consider the Miller-Spencer (MS) [44], AV18 [45], and CD-Bonn [46, 47] parameterizations.
TABLE 3: $0 \nu \beta \beta$ NMEs values for SUSY2 mechanism and comparison to other results in the literature.

\begin{tabular}{lcccc}
\hline$M^{0 v}$ & No SRC & Jastrow & AV-18 & CD-BONN \\
\hline${ }^{48} \mathrm{Ca}(\mathrm{GXPF1A})$ & 65.1 & 46.4 & 65.0 & 70.0 \\
${ }^{48} \mathrm{Ca}(\mathrm{KB} 3 \mathrm{G})$ & 72.9 & 51.8 & 72.9 & 78.6 \\
${ }^{48} \mathrm{Ca}[34]$ & & & 81.8 & 86.7 \\
${ }^{76} \mathrm{Ge}(\mathrm{JUN} 45)$ & 281.7 & 233.4 & 283.5 & 296.8 \\
${ }^{76} \mathrm{Ge}[32]$ & & & 594 & 612 \\
${ }^{82} \mathrm{Se}(\mathrm{JUN} 45)$ & 253.6 & 208.6 & 255.7 & 268.1 \\
${ }^{82} \mathrm{Se}[32]$ & & & 578 & 595 \\
\hline
\end{tabular}

\section{Numerical Results and Discussions}

We calculate first the NMEs for the three nuclei, that is, ${ }^{48} \mathrm{Ca}$, ${ }^{76} \mathrm{Ge}$, and ${ }^{82} \mathrm{Se}$, and the three mechanisms mentioned above, using the code described in detail in $[35,36]$. For ${ }^{48} \mathrm{Ca}$, we performed calculations with two different $\mathrm{NN}$ interactions, GXPF1A [51] and KB3G [52] interactions, while for the other two isotopes we used the JUN45 [53] NN interaction. In the case of ${ }^{48} \mathrm{Ca}$, our model space includes the whole $\mathrm{fp}$ shell $f_{7 / 2}, p_{3 / 2}, f_{5 / 2}$, and $p_{1 / 2}$, while, for ${ }^{76} \mathrm{Ge}$ and ${ }^{82} \mathrm{Se}$, the model space is jj44, including the following orbitals $f_{5 / 2}$, $p_{3 / 2}, p_{1 / 2}$, and $g_{9 / 2}$. For the nuclear parameters involved in calculations, we used the values mentioned in the previous section and, for the nuclear radius, $R=r_{0} A^{1 / 3}$, we used $r_{0}=1.2 \mathrm{fm}$. The results are presented in Tables 1,2 , and 3 . One can see that there are significant differences between the results when using different $\mathrm{NN}$ interactions and different SRC parameterizations. Indeed, especially for the short-range exchange mechanisms, as the heavy neutrino and gluino (SUSY1) ones, one expects the results to be sensitive to the $\mathrm{NN}$ interaction and to the type of SRC parameterizations. The uncertainties in the NME calculated values due to the use of different $\mathrm{NN}$ interactions are in the range of (10$16) \%$. The uncertainties due to the use of different SRC parameterizations are larger; sometimes differences between results are larger than $100 \%$, especially when comparing the NME values obtained with MS [44] parameterizations with those obtained with softer parameterizations, such as AV18 [45] and CD-Bonn [46, 47]. Further, we compare our results with other similar ones found in literature. To the best of our knowledge, explicit values of NMEs calculated with large scale ShM approaches are reported only in [34] and for one nucleus, ${ }^{48} \mathrm{Ca}$. Our results agree within $\sim 10 \%$ with those from [34], for all three mechanisms. At this point, it is worth mentioning that the GT and tensor contributions in (5) must have a relative opposite sign, such that the adding of the tensor contribution decreases the $M^{0 v}$ total value. Hence, one has to pay attention when applying formula (5) because the GT and tensor contributions can come out from numerical calculations with opposite signs. In this respect, we suspect that our NME values agree even better with those from [34]. For the other two isotopes, that is, ${ }^{76} \mathrm{Ge}$ and ${ }^{82} \mathrm{Se}$, we compare our (ShM) results with QRPA $[31,32,43]$ and IBM-2 [28] results. For the heavy neutrino and SUSY2 mechanisms, the differences between our NME values and NME values 
TABLE 4: Upper limits for Majorana neutrino mass parameters together with the other components of the $0 \nu \beta \beta$ decay halftimes: the experimental lifetimes lower limits, the phase space factors, and the nuclear matrix elements.

\begin{tabular}{ccccccccc}
\hline & $T_{\exp }^{0 \nu}[\mathrm{yr}]$ & $G^{0 \nu}\left[\mathrm{yr}^{-1}\right]$ & $M_{N}^{0 \nu}$ & $M_{\lambda^{\prime}}^{0 \nu}$ & $M_{q}^{0 \nu}$ & $\left\langle\eta_{N}\right\rangle$ & $\left\langle\eta_{\lambda^{\prime}}\right\rangle$ \\
\hline${ }^{48} \mathrm{Ca}^{*}$ & $5.8 \cdot 10^{22}[48]$ & $2.46 E-14$ & 70.3 & 548.2 & 70 & $2.42 \cdot 10^{-7}$ & $3.10 \cdot 10^{-8}$ & $2.42 \cdot 10^{-7}$ \\
${ }^{48} \mathrm{Ca}^{\dagger}$ & $5.8 \cdot 10^{22}[48]$ & $2.46 E-14$ & 82.8 & 641.7 & 78.6 & $2.05 \cdot 10^{-7}$ & $2.64 \cdot 10^{-8}$ & $2.16 \cdot 10^{-7}$ \\
${ }^{76} \mathrm{Ge}$ & $2.1 \cdot 10^{25}[49]$ & $2.37 E-15$ & 199.2 & 1509.4 & 296.8 & $0.14 \cdot 10^{-7}$ & $0.19 \cdot 10^{-8}$ & $0.10 \cdot 10^{-7}$ \\
${ }^{82} \mathrm{Se}$ & $3.6 \cdot 10^{23}[50]$ & $1.01 E-14$ & 184.5 & 1393.5 & 268.1 & $0.58 \cdot 10^{-7}$ & $0.76 \cdot 10^{-8}$ & $0.40 \cdot 10^{-7}$ \\
\hline
\end{tabular}

${ }^{*}$ Denotes GXPF1A [51] effective interaction and ${ }^{\dagger} \mathrm{KB} 3 \mathrm{G}[52]$ effective interaction.

calculated with QRPA and IBM-2 methods are significant, but they are similar (in magnitude) to those encountered usually in the case of light neutrino exchange mechanism. For the SUSY1 mechanism, our results compare differently with the QRPA results reported by Tuebingen group in $[32,43]$, the only ones found in literature. We note that the agreement is good when comparing with the results of [43], where the MS parameterizations of the SRCs are employed, and it is bad when comparing with those of [32], where AV18 and CDBonn parameterizations for SRCs are used. As a conclusion, it turns out that more results for the NMEs associated with heavy neutrino and SUSY mechanisms, performed with different methods and including more nuclei, are needed for a relevant analysis of their validity.

In Table 4, we present new upper limits for the LNV parameters associated with the studied $0 \nu \beta \beta$ decay mechanisms, in the hypothesis of a single dominance mechanism. The PSFs values are taken from [37] and we mention that they are very close to PSF values reported in $[38,39]$. The PSFs have been calculated since long time $[40,41]$ but they were less discussed, being considered to be computed with enough precision. Recently, they were recalculated with improved approaches, by using exact electron Dirac wave functions, taking into account the finite nuclear size and electron screening effects [38] and, in addition, a realistic Coulomb potential $[39,42]$, and differences/discrepancies were found in several cases between the old and the recent PSF values. For the three isotopes considered in this work, the new PSF values differ from older calculations (e.g., from those reported in $[41,54-56])$ up to $10 \%$, justifying hence their use in a precise derivation of LNV parameters. The uncertainties in NME values reflect on the precision of derivation the LNV parameters. According to the consensus agreed upon in literature that softer SRC parameterizations are indicated, we adopt the NME values calculated with the CD-Bonn parameterization and derive the LNV parameters for the three nuclei and the three mechanisms considered. These results are presented in Table 4. For derivation, we used the most recent results for the experimental lifetimes, reported in references indicated in parenthesis. For ${ }^{48} \mathrm{Ca}$, our limits for the LNV parameters are close to those reported in [34], while for the other two nuclei they differ from other results from literature [28, 32, 34, 43], derived with NMEs values calculated with QRPA and IBM-2 methods. As we already mentioned, information on LNV parameters for the heavy neutrino mechanism can be now extracted, as well, from the data analysis of the same sign dilepton channels at the LHC experiments. Thus, we expect that complementary information from both low- and high-energy experiments could be used in the future for better constraining of the LNV parameters.

\section{Conclusions}

We report new constraints for the heavy neutrino and SUSY parameters derived from an analysis of $0 \nu \beta \beta$ decay of three experimentally interesting nuclei, ${ }^{48} \mathrm{Ca},{ }^{76} \mathrm{Ge}$, and ${ }^{82} \mathrm{Se}$. To the best of our knowledge, for the last two isotopes, no other large scale ShM results for these mechanisms are available so far. For deduction of these parameters, precise values of the NMEs and PSFs which enter the lifetime formulae are needed, as well as accurate experimental measurements of the lifetimes. The NME calculations are performed with a recent ShM code described widely in $[35,36]$. The PSFs are taken from our most recent calculations reported in [37]. For SUSY mechanisms, we discuss two mechanisms associated with possible R-parity breaking in SUSY theories, a short-range one with exchange of heavy Majorana neutrino and scalar SUSY particles (gluinos and squarks and/or neutralinos and selectrons) and a long-range one involving exchange of both heavy squarks and light Majorana neutrinos-"squarkneutrino" mechanism. First, we calculated the NMEs using different nuclear ingredients. For ${ }^{48} \mathrm{Ca}$, we use two different $\mathrm{NN}$ interactions, GXPF1A and KB3G, and found differences between the results within $10 \%$. Also, we performed the calculations using three different parameterizations for the SRCs and found a significant dependence of the results on the way the SRCs are introduced in calculations. Further, we compare our (ShM) results with similar results from literature. For ${ }^{48} \mathrm{Ca}$, our results are in good agreement with the results from [34], for all three mechanisms. For the other two isotopes, ${ }^{76} \mathrm{Ge}$ and ${ }^{82} \mathrm{Se}$, we compare our (ShM) NMEs with those computed by QRPA [31, 32, 43] and IBM-2 [28] methods. For the heavy neutrino and SUSY2 mechanisms, the differences between our results and the results from those references are significant but similar (up to a factor of two) to those encountered usually in literature in the case of light neutrino exchange mechanism. For the SUSY1 mechanism, our results compare differently with QRPA results, reported by Tuebingen group. There is a good agreement with the results from [43], when the MS SRC parameterization is employed, but the agreement is bad when comparing with the values from [32], when softer SRC parameterizations are employed. As a conclusion, it turns out that more results for the NMEs associated with these $0 \nu \beta \beta$ decay mechanisms, alternative to the light neutrino exchange one, performed with different nuclear methods and including more nuclei, 
are needed for a relevant analysis of their validity. Then, we derived upper limits of the LNV parameters associated with the three mechanisms, in the hypothesis of one single dominance mechanism, using NMEs values computed with CD-Bonn parameterization. Finally, it is worth noting that information on LNV parameters can now be provided by LHC experiments at CERN from the analysis of the same sign dilepton channels. Thus, there is now the possibility to gather complementary information from both low- and high-energy experiments and use it to better constrain LNV parameters.

\section{Conflict of Interests}

The authors declare that there is no conflict of interests regarding the publication of this paper.

\section{Acknowledgments}

The authors thank J. Menendez for useful discussions. This work was done with the support of the MEN and UEFISCDI through the Project IDEI-PCE-3-1318, Contract no. $58 / 28.10 / 2011$.

\section{References}

[1] F. T. Avignone, S. R. Elliott, and J. Engel, "Double beta decay, Majorana neutrinos, and neutrino mass," Reviews of Modern Physics, vol. 80, no. 2, pp. 481-516, 2008.

[2] H. Ejiri, "Double beta decays and neutrino nuclear responses," Progress in Particle and Nuclear Physics, vol. 64, no. 2, pp. 249257, 2010.

[3] J. D. Vergados, H. Ejiri, and F. Šimkovic, "Theory of neutrinoless double-beta decay," Reports on Progress in Physics, vol. 75, no. 10, Article ID 106301, 2012.

[4] W. Rodejohann, "Neutrinoless double-beta decay and neutrino physics," Journal of Physics G: Nuclear and Particle Physics, vol. 39, no. 12, Article ID 124008, 2012.

[5] A. Faessler, V. Rodin, and F. Simkovic, "Nuclear matrix elements for neutrinoless double-beta decay and double-electron capture," Journal of Physics G: Nuclear and Particle Physics, vol. 39, no. 12, Article ID 124006, 2012.

[6] P. Vogel, "Nuclear structure and double beta decay," Journal of Physics G: Nuclear and Particle Physics, vol. 39, no. 12, Article ID 124002, 2012.

[7] R. N. Mohapatra and J. C. Pati, "Left-right gauge symmetry and an "isoconjugate \& model of CP violation," Physical Review D, vol. 11, no. 3, pp. 566-571, 1975.

[8] M. Doi, T. Kotani, H. Nishiura, and E. Takasugia, "Double beta decay," Progress of Theoretical Physics, vol. 69, no. 2, pp. 602-635, 1983.

[9] M. Hirsch, H. V. Klapdor-Kleingrothaus, and S. G. Kovalenko, "Supersymmetry and neutrinoless double beta decay," Physical Review D, vol. 53, no. 3, pp. 1329-1348, 1996.

[10] CMS Collaboration, "Search for new physics with same-sign isolated dilepton events with jets and missing transverse energy at the LHC," Journal of High Energy Physics, vol. 2011, article 77, 2011.

[11] ATLAS Collaboration, "Inclusive search for same-sign dilepton signatures in pp collisions at $\sqrt{S}=7 \mathrm{TeV}$ with the ATLAS detector," Journal of High Energy Physics, vol. 2011, article 107, 2011.

[12] LHCb Collaboration, "Search for Lepton number violating decays $B^{+} \rightarrow \pi^{-} \mu^{+} \mu^{+}$and $B^{+} \rightarrow K^{-} \mu^{+} \mu^{+}$," Physical Review Letters, vol. 108, Article ID 101601, 2012.

[13] LHCb Collaboration, "Searches for Majorana neutrinos in $\mathrm{B}^{-}$ decays," Physical Review D, vol. 85, p. 112004, 2012.

[14] F. Simkovic, A. Faessler, H. Muther, V. Rodin, and M. Stauf, " $0 \nu \beta \beta$-decay nuclear matrix elements with self-consistent short-range correlations," Physical Review C, vol. 79, Article ID 055501, 2009.

[15] V. A. Rodin, A. Faessler, F. Simkovic, and P. Vogel, "Uncertainty in the $0 v \beta \beta$ decay nuclear matrix elements ," Physical Review $C$, vol. 68, Article ID 044302, 2003.

[16] V. A. Rodin, A. Faessler, F. Simkovic, and P. Vogel, "Assessment of uncertainties in QRPA $0 \nu \beta \beta$-decay nuclear matrix elements," Nuclear Physics A, vol. 766, pp. 107-131, 2006.

[17] V. A. Rodin, A. Faessler, F. Simkovic, and P. Vogel, "Erratum to: "Assessment of uncertainties in QRPA $0 \nu \beta \beta$-decay nuclear matrix elements" [Nucl. Phys. A 766 (2006) 107]," Nuclear Physics A, vol. 793, no. 1-4, pp. 213-215, 2007.

[18] M. Kortelainen and J. Suhonen, "Nuclear matrix elements of $0 \nu \beta \beta$ decay with improved short-range correlations," Physical Review C, vol. 76, Article ID 024315, 2007.

[19] F. Šimkovic, A. Faessler, V. Rodin, P. Vogel, and J. Engel, "Anatomy of the $0 \nu \beta \beta$ nuclear matrix elements," Physical Review C, vol. 77, no. 4, Article ID 045503, 2008.

[20] S. Stoica and H. V. Klapdor-Kleingrothaus, "Critical view on double-beta decay matrix elements within Quasi Random Phase Approximation-based methods," Nuclear Physics A, vol. 694, no. 1-2, pp. 269-294, 2001.

[21] M. Horoi and S. Stoica, "Shell model analysis of the neutrinoless double- $\beta$ decay of ${ }^{48} \mathrm{Ca}$," Physical Review $C$, vol. 81, no. 2 , Article ID 024321, 2010.

[22] E. Caurier, A. P. Zuker, A. Poves, and G. Martinez-Pinedo, "Full pf shell model study of $A=48$ nuclei," Physical Review $C$, vol. 50, article 225, 1994.

[23] J. Retamosa, E. Caurier, and F. Nowacki, "Neutrinoless double beta decay of ${ }^{48} \mathrm{Ca}$," Physical Review C, vol. 51, article 371, 1995.

[24] E. Caurier, J. Menéndez, F. Nowacki, and A. Poves, "Influence of pairing on the nuclear matrix elements of the neutrinoless $\beta \beta$ decays," Physical Review Letters, vol. 100, no. 5, Article ID 052503, 2008.

[25] J. Menéndez, A. Poves, E. Caurier, and F. Nowacki, "Disassembling the nuclear matrix elements of the neutrinoless $\beta \beta$ decay," Nuclear Physics A, vol. 818, no. 3-4, pp. 139-151, 2009.

[26] J. Barea and F. Iachello, "Neutrinoless double- $\beta$ decay in the microscopic interacting boson model," Physical Review $C$, vol. 79, no. 4, Article ID 044301, 2009.

[27] J. Barea, J. Kotila, and F. Iachello, "Limits on neutrino masses from neutrinoless double- $\beta$ decay," Physical Review Letters, vol. 109, no. 4, Article ID 014315, 2012.

[28] J. Barea, J. Kotila, and F. Iachello, "Nuclear matrix elements for double- $\beta$ decay," Physical Review C, vol. 87, Article ID 014315, 2013.

[29] P. K. Rath, R. Chandra, K. Chaturvedi, P. K. Raina, and J. G. Hirsch, "Uncertainties in nuclear transition matrix elements for neutrinoless $\beta \beta$ decay within the projected-Hartree-FockBogoliubov model," Physical Review C, vol. 82, no. 6, Article ID 064310, 2010. 
[30] T. R. Rodriguez and G. Martinez-Pinedo, "Energy density functional study of nuclear matrix elements for neutrinoless $\beta \beta$ Decay," Physical Review Letters, vol. 105, Article ID 252503, 2010.

[31] F. Šimkovic, G. Pantis, J. D. Vergados, and A. Faessler, "Additional nucleon current contributions to neutrinoless double $\beta$ decay," Physical Review C, vol. 60, no. 5, Article ID 055502, 1999.

[32] A. Faessler, G. L. Fogli, E. Lisi, A. M. Rotunno, and F. Åimkovic, "Multi-isotope degeneracy of neutrinoless double- $\beta$ decay mechanisms in the quasiparticle random-phase approximation," Physical Review D, vol. 83, no. 11, Article ID 113015, 2011.

[33] M. Blennow, E. Fernandez-Martinez, J. Lopez-Pavon, and J. Menendez, "Neutrinoless double beta decay in seesaw models," Journal of High Energy Physics, vol. 2010, article 96, 2010.

[34] M. Horoi, "Shell model analysis of competing contributions to the double- $\beta$ decay of ${ }^{48}$ Ca," Physical Review C, vol. 87, Article ID 014320, 2013.

[35] A. Neacsu, S. Stoica, and M. Horoi, "Fast, efficient calculations of the two-body matrix elements of the transition operators for neutrinoless double- $\beta$ decay," Physical Review $C$, vol. 86, no. 6 , Article ID 067304, 2012.

[36] A. Neacsu and S. Stoica, "Study of nuclear effects in the computation of the $0 v \beta \beta$ decay matrix elements," Journal of Physics G: Nuclear and Particle Physics, vol. 41, no. 1, Article ID 015201, 2014.

[37] S. Stoica and A. Neacsu, "Constraints on light neutrino parameters derived from the study of neutrinoless double beta decay," Advances in High Energy Physics, vol. 2014, Article ID 745082, 7 pages, 2014.

[38] J. Kotila and F. Iachello, "Phase-space factors for double- $\beta$ decay," Physical Review C, vol. 85, no. 3, Article ID 034316, 2012.

[39] S. Stoica and M. Mirea, "New calculations for phase space factors involved in double- $\beta$ decay," Physical Review $C$, vol. 88, Article ID 037303, 2013.

[40] H. Primakoff and S. P. Rosen, "Double beta decay," Reports on Progress in Physics, vol. 22, no. 1, article 305, pp. 121-166, 1959.

[41] J. Suhonen and O. Civitarese, "Weak-interaction and nuclearstructure aspects of nuclear double beta decay," Physics Report, vol. 300, no. 3-4, pp. 123-214, 1998.

[42] T. E. Pahomi, A. Neacsu, M. Mirea, and S. Stoica, "Phase space calculations for beta- beta- decays to final excited $2+1$ states," Romanian Reports in Physics, vol. 66, no. 2, pp. 370-375, 2014.

[43] A. Wodecki, W. A. Kaminski, and F. Simkovic, "Grand unified theory constrained supersymmetry and neutrinoless double $\beta$ decay," Physical Review D, vol. 60, Article ID 115007, 1999.

[44] T. Tomoda, "Double beta decay," Reports on Progress in Physics, vol. 54, no. 1, pp. 53-126, 1991.

[45] C. Giusti, H. Müther, F. D. Pacati, and M. Stauf, "Short-range and tensor correlations in the $16 \mathrm{O}\left(\mathrm{e}, \mathrm{e}^{\prime} \mathrm{pn}\right)$ reaction," Physical Review C, vol. 60, no. 5, Article ID 054608, 1999.

[46] H. Muther and A. Polls, "Correlations derived from modern nucleon-nucleon potentials," Physical Review C, vol. 61, Article ID 014304, 1999.

[47] H. Muther, "Two-body correlations in nuclear systems," Progress in Particle and Nuclear Physics, vol. 45, no. 1, pp. 243334, 2000.

[48] S. Umehara, T. Kishimoto, I. Ogawa et al., "Neutrino-less double- $\beta$ decay of ${ }^{48} \mathrm{Ca}$ studied by $\mathrm{CaF}_{2}(\mathrm{Eu})$ scintillators," Physical Review C, vol. 78, Article ID 058501, 2008.

[49] C. Macolino and GERDA Collaboration, "Results on neutrinoless double-beta decay from GERDA phase I," Modern Physics Letters A, vol. 29, no. 1, p. 1430001, 2014.
[50] A. S. Barabash and V. B. Brudanin, "Investigation of double-beta decay with the NEMO-3 detector," Physics of Atomic Nuclei, vol. 74, no. 2, pp. 312-317, 2011.

[51] M. Honma, T. Otsuka, B. A. Brown, and T. Mizusaki, "New effective interaction for $p f$ nuclei and its implications for the stability of the $N=Z=28$ closed core," Physical Review $C$, vol. 69, no. 3, Article ID 034335, 2004.

[52] T. T. S. Kuo and G. E. Brown, "Reaction matrix elements for the 0f-1p shell nuclei," Nuclear Physics A, vol. 114, no. 2, pp. 241-279, 1968.

[53] M. Honma, T. Otsuka, T. Mizusaki, and M. Hjorth-Jensen, "New effective interaction for $f_{5} p g_{9}$-shell nuclei," Physical Review C, vol. 80, no. 6, Article ID 064323, 2009.

[54] M. Doi, T. Kotani, and E. Takasugi, "Double beta decay and majorana neutrino," Progress of Theoretical Physics Supplements, vol. 83, pp. 1-175, 1985.

[55] M. Doi and T. Kotani, "Neutrino emitting modes of double beta decay," Progress of Theoretical Physics, vol. 87, no. 5, pp. 12071231, 1992.

[56] M. Doi and T. Kotani, "Neutrinoless modes of double beta decay," Progress of Theoretical Physics, vol. 89, pp. 139-159, 1993. 

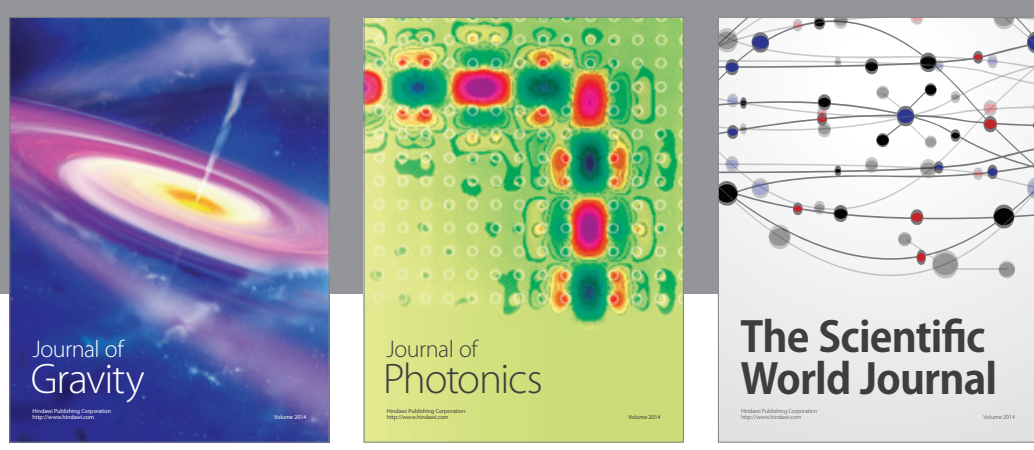

The Scientific World Journal
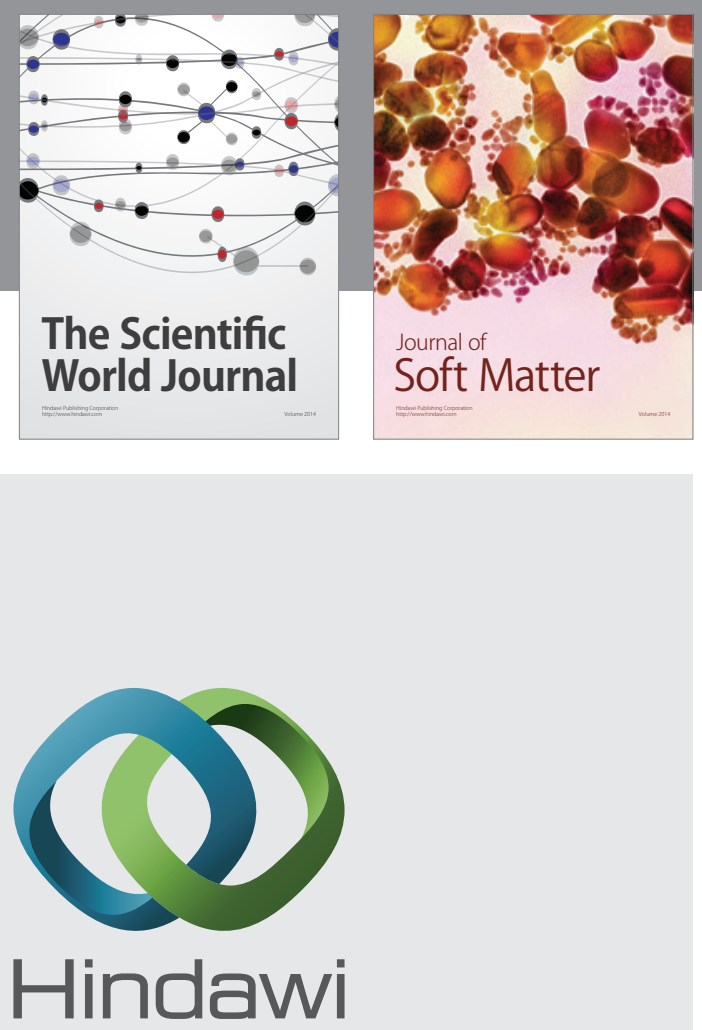

Submit your manuscripts at

http://www.hindawi.com

nternational Journal of

Statistical Mechanics
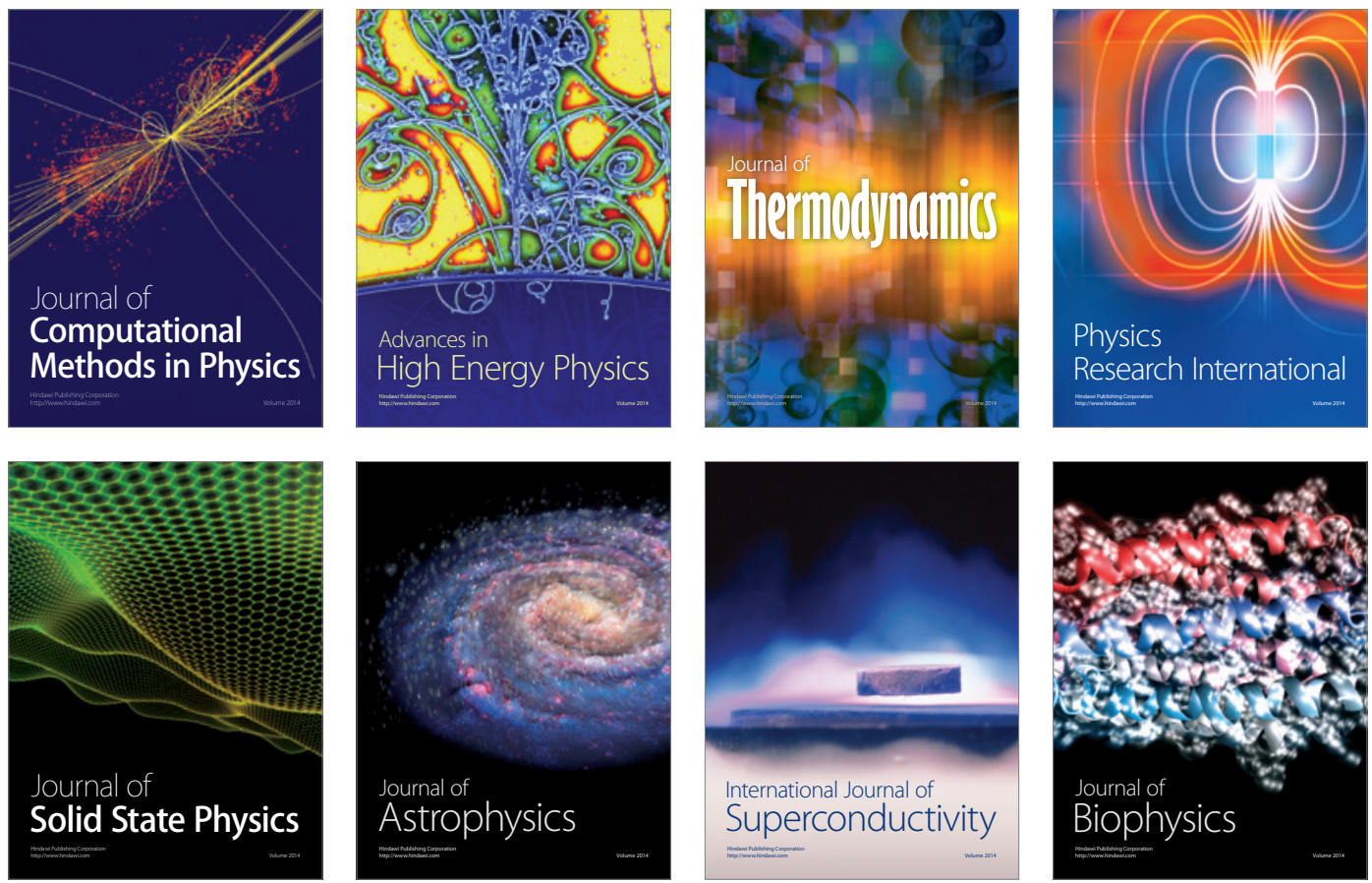
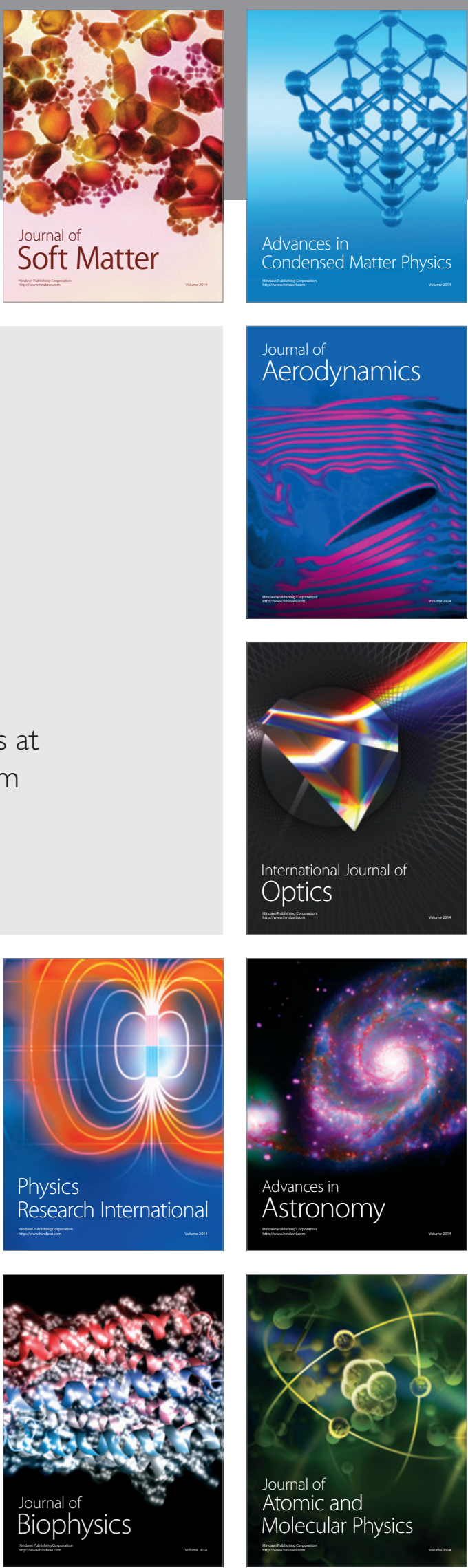